\title{
Efeito de uma história de FR sobre a taxa de respostas em FI e DRL*
} Effects of a FR history on responses rate in FI and DRL

\section{Efecto de una historia de FR en la tasa de respuesta en FI y DRL}

Carlos Eduardo Costa ${ }^{1}$, Raquel Fernanda Ferreira Lacerda ${ }^{2}$, Lucas Roberto Pedrão Paulino ${ }^{3}$, Roberto Alves Banaco ${ }^{4}$

[1] [2] Universidade Estadual de Londrina [3] Universidade de São Paulo [4] Núcleo Paradigma; Pontifícia Universidade Católica, SP I Título abreviado: História e persistência comportamental I Endereço para correspondência: Universidade Estadual de Londrina. Centro de Ciências Biológicas. Departamento de Psicologia Geral e Análise do Comportamento. Caixa Postal: 10.011. CEP 86057-970. Londrina/PR I Email: caecosta@uel.br I robertobanaco@nucleoparadigma.com.br

Resumo: Realizaram-se dois experimentos para verificar se altas taxas de respostas, selecionadas por FR, seriam igualmente afetadas quando o programa de reforço mudasse para FI e para DRL. Universitários foram expostos à seguinte sequência de programas de reforço: FR-FI-FR-DRL. No Experimento 1, cinco participantes foram expostos a três sessões de 30 min. e cada programa foi correlacionado com diferentes cores do botão de respostas. No Experimento 2, quatro participantes foram expostos a seis sessões de $30 \mathrm{~min}$. em cada programa de reforço, sem mudança na cor do botão de respostas. Nos dois experimentos, as taxas de respostas foram relativamente altas para todos os participantes durante o FR. Quando o programa de reforço mudou para FI, a taxa de respostas tendeu a diminuir para quatro dos nove participantes e quando o programa mudou para DRL, as taxas de respostas diminuíram para todos os participantes. Esses resultados não foram afetados pelo fato de os programas de reforço serem correlacionados (Experimento 1) ou não (Experimento 2) com diferentes estímulos, exceto nos minutos iniciais da exposição ao FI.

Palavras-chave: história comportamental, persistência comportamental, esquemas de reforçamento, sensibilidade, humanos.

* Projeto de pesquisa financiado pela Fundação Araucária (Programa de Apoio à Pesquisa Básica e Aplicada - Chamada 02/2006 - Protocolo 8648; Convênio UEL no 35/2007). Raquel F. F. Lacerda foi bolsista IC/UEL, Fundação Araucária e PIBIC/CNPq-UEL durante a realização deste trabalho. 
Abstract: Two experiments were conducted to assess if high response rates, selected by FR, would be equally affected when the schedule of reinforcement changed to FI and DRL. Undergraduate students were exposed to the following sequence of reinforcement schedules: FR-FI-FR-DRL. In Experiment 1, five participants were exposed to each schedule for three, 30 min, sessions and each schedule was correlated with a different color of the response button. In Experiment 2, four participants were exposed to each schedule for six, $30 \mathrm{~min}$, sessions and the color of the response button was the same across schedules. In both experiments, response rates were high for all the participants under the FR. When the schedule of reinforcement changed to an FI, (a) response rates decreased for four of nine participants; (b) when the schedule changed to a DRL, response rates decreased for all participants. These results were not affected by the schedules being correlated (Experiment 1) or not (Experiment 2) with different stimuli, except under the initial minutes of exposure to the FI schedule.

Keywords: behavioral history, behavioral persistence, schedules of reinforcement, sensitivity, humans.

Resumen: Se realizaron dos experimentos para comprobar si las altas tasas de respuestas, elegidas por RF, también se verían afectadas cuando el programa de refuerzo cambia a FI y DRL. Estudiantes universitarios fueron expuestos a la siguiente secuencia de los programas de refuerzo: FR-FI-FR-DRL. En el Experimento 1, los cinco participantes fueron expuestos a tres sesiones de 30 minutos y cada programa fue correlacionado con distintos colores del botón. En el Experimento 2, cuatro participantes fueron expuestos a seis sesiones de $30 \mathrm{~min}$ en cada programa, sin cambio de color del botón. En los dos experimentos, las tasas de respuestas fueron relativamente altos a todos los participantes durante la RF. Cuando el programa de refuerzo ha cambiado a FI, la tasa de respuestas tendió a disminuir a cuatro de los nueve participantes y cuando el programa cambió a DRL, han disminuido las tasas de respuesta para todos los participantes. Estos resultados no fueron afectados por el hecho de que los programas de fortalecimiento ser correlacionados (Experimento 1) o no (Experimento 2) con diferentes estímulos, excepto la exposición temprana a FI.

Palabras-clave: Persistencia del comportamiento, programas reforzamiento, comportamiento regido por reglas, humanos. 
Uma questão importante para a Psicologia como um todo e para a Análise do Comportamento em particular, diz respeito à "persistência" do comportamento (cf. Weiner, 1970), ao longo do tempo, frente a mudanças que ocorrem na vida de uma pessoa. Que variáveis podem aumentar ou diminuir a probabilidade de um comportamento persistir quando as contingências mudam? Diversas pesquisas têm sido realizadas buscando, de alguma maneira, responder a essa questão (e.g., Cohen, Riley, \& Weigle, 1993; Hayes, Brownstein, Haas, \& Greenway, 1986; Kaufman, Baron, \& Kopp, 1966; Mace, Mauro, Boyajian, \& Eckert, 1997; Weiner, 1969). Essas pesquisas têm sido desenvolvidas em áreas de estudos aparentemente diversas, dentro da Análise do Comportamento, tais como momentum comportamental (e.g., dos Santos, 2005; Nevin, Mandell, \& Atak, 1983), história comportamental (e.g., Aló, 2005; Costa, Cirino, Cançado, \& Soares, 2009; Wanchisen, 1990) e controle instrucional (e.g., Albuquerque, 2001; Matos, 2001; Skinner, 1969, 1974).

Em um estudo que buscava identificar variáveis que pudessem contribuir para a persistência comportamental em humanos, Costa et al. (2008) expuseram participantes a uma de duas histórias de reforço (Razão Fixa [FR] ou Reforço Diferencial de Baixas Taxas [DRL]) com um de três tipos de reforços (pontos trocados por dinheiro, pontos trocados por fotocópias ou pontos apenas). Em seguida, todos os participantes foram expostos a sessões em Intervalo Fixo (FI), mantido o tipo de reforço empregado na condição anterior. Durante a exposição ao FI, a taxa de respostas de pressionar um botão se manteve similar à condição anterior (i.e., altas taxas para participantes com história em FR e baixas taxas para participantes com história em DRL) quando a consequência programada foi ou pontos trocados por dinheiro ou pontos trocados por fotocópias, mas o efeito da história desapareceu mais rapidamente quando apenas os pontos obtidos na sessão foram empregados como reforçadores. Altas taxas de respostas em FI, após uma história de exposição ao FR, também foram observadas nos estudos de Weiner $(1964,1969)$ - com humanos e LeFrancois e Metzger (1993) - com ratos. Além disso, Mace et al. (1997) também observaram que o tipo do reforçador pode afetar a resistência do comportamento à mudança (em humanos e ratos).
Esses resultados podem sugerir que, após a exposição a uma determinada história de reforço e, eventualmente, quando certo tipo de reforçador é empregado, torna-se mais provável que o comportamento de humanos fique "insensível" à mudança nas contingências de reforço - uma interpretação comum em vários estudos sobre controle instrucional (e.g., Hayes, Brownstein, Zettle, et al., 1986; Lowe, 1979; Shimoff, Catania, \& Matthews, 1981). Todavia, uma característica importante do responder em FI é que ele permite ampla variação na taxa de respostas sem afetar a taxa de reforço. No caso de Costa et al. (2008), os participantes continuaram a receber todos os pontos programados no experimento, quer as taxas de respostas fossem altas ou baixas.

Galizio (1979, Experimento 2), em um estudo que envolvia controle instrucional, observou que a manutenção ou não de um desempenho estava relacionada à manutenção dos reforçadores. Seis universitários foram expostos a um procedimento de esquiva não sinalizada. Na Fase 1, todos os participantes foram expostos a um programa múltiplo de reforço com instruções correspondentes (pressionar o botão a cada $t$ segundos) para cada componente (o valor de $t$ era diferente em cada componente). Na Fase 2, as instruções permaneciam inalteradas, porém o programa de reforço mudava para um múltiplo em que a perda de pontos era "desligada"; se o participante seguisse a instrução do experimentador, ele não entraria em contato com a discrepância entre a instrução e a nova contingência programada. Na Fase 3, a instrução permanecia inalterada e o programa de reforço mudava para um múltiplo em que, caso o participante seguisse as instruções, ele entraria em contato com a discrepância entre as instruções e a contingência (i.e., perderia pontos). Os resultados indicaram que, quando o seguimento das instruções não produziu perda de pontos (Fase 2), os participantes mantiveram as taxas de respostas descritas pela instrução. Entretanto, quando o seguimento das instruções produziu perda de pontos (Fase 3), os participantes a abandonaram.

Resumidamente, os resultados de Costa et al. (2008) sugeriram que, quando o programa de reforço é alterado, as taxas de respostas tendem a ser mantidas se o ganho de pontos é mantido e se os 
pontos são trocados por dinheiro enquanto o estudo de Galizio (1979) sugeriu que as taxas de respostas tendem a ser mantidas se isso não implicar na perda de pontos e tendem a mudar se a manutenção da taxa de respostas implicar na perda de reforçadores.

Resultados indicando mudança do responder quando a manutenção do desempenho produzia perda de reforçadores foram encontrados também por Hunziker, Caramori, Silva e Barba (1998), Costa, Soares, Becker e Banaco (2009) e Costa, de Souza, Bianchini, Porto e de Freitas (2013). Por exemplo, Costa, Soares et al. expuseram 10 participantes aos seguintes programas de reforço, nesta ordem: FR e FI-custo 1 (perda de um ponto para cada resposta emitida durante o intervalo entre reforços), FR e FI-custo 10 (perda de 10 pontos para cada resposta emitida durante o intervalo). Quando o programa de reforço era cumprido, 100 pontos eram creditados em um contador. Os resultados indicaram que, quando a contingência mudou de FR para FI-custo, as taxas de respostas tenderam a diminuir em relação ao FR, independentemente do valor do custo da resposta. Entretanto, foram alterados simultaneamente dois aspectos da contingência: o programa de reforço (de FR para FI) e a introdução do custo da resposta no FI.

$\mathrm{O}$ que aconteceria com o comportamento se o arranjo experimental das três primeiras fases de Costa et al. (2008) fosse mantido (i.e., FR - FI sem custo), em seguida a linha de base em FR fosse retomada e, posteriormente, o programa de reforço mudasse para DRL, no qual a manutenção de altas taxas de respostas (possivelmente selecionadas durante a história de FR) não produzisse ganho de pontos? O procedimento do presente trabalho, elaborado a partir desta pergunta, procurou investigar como a manutenção ou não de reforçadores (cf. Galizio, 1979; Paracampo \& Albuquerque, 2004) afetaria a persistência de um padrão comportamental selecionado na história (cf. Costa et al., 2008; Costa, Soares et al., 2009; Weiner, 1969). Adicionalmente investigou-se o efeito da mudança de estímulos correlacionada com cada programa de reforço utilizado (Experimento 1 vs. Experimento 2).

Apesar de existirem estudos que sugerem que a taxa de respostas tende a se manter inalterada quando ocorre a transição do FR para o FI (e.g.,
Weiner, 1964, 1969, 1970; ver Costa et al., 2008, para resultados discrepantes) ou que a taxa diminui quando a transição é de FR para DRL (e.g., Cole, 2001; Wanchisen, Sutphin, Balogh, \& Tatham, 1998; Weiner, 1969), há resultados que poderiam levantar dúvidas e sugerem que um experimento que manipule diretamente estes programas de reforço (FR para FI e FR para DRL) merece ser empreendido.

Em primeiro lugar, embora estudos tenham indicado que as taxas de respostas em FI tendem a diminuir quando há custo para a resposta (perda de pontos), após história de exposição a um FR (e.g., Costa, Soares et al., 2009; Costa, de Souza et al.; 2013), estudos anteriores haviam indicado que as taxas de respostas em FI tendiam a se manter altas após história de FR, mesmo que a contingência de FI envolvesse custo (e.g., Weiner, 1965, 1969, 1970). Em segundo lugar, há estudos que sugerem que humanos são "insensíveis" (i.e., não emitem taxas de respostas diferenciadas) em um programa múltiplo FR DRL (e.g., Hayes, Brownstein, Zettle, Rosenfarb, \& Korn, 1986; Hayes, Brownstein, Haas et al., 1986; Rosenfarb, Newland, Brannon, \& Howey, 1992; Wulfert, Greenway, Farkas, Hayes, \& Dougher, 1994), sugerindo que baixas taxas de respostas nem sempre são obtidas em transições entre FR e DRL (ver Porto, Ramos, \& Costa, 2011 sobre a questão da insensibilidade de humanos a programas de reforço múltiplo FR DRL). Por fim, os experimentos de Weiner (1969) sugerem que o comportamento se mantém mais com a mudança de FR para FI do que de FR para DRL, mas essa conclusão é retirada da comparação de dados entre participantes e não em um delineamento de sujeito único - do tipo ABAC -, como o realizado no presente experimento.

\section{Experimento 1}

O objetivo foi investigar o efeito da alteração do programa de reforço de FR para FI e de FR para DRL sobre a as taxas de respostas quando um estímulo (a cor do botão de resposta) foi correlacionado com cada programa de reforço. 


\section{Método}

\section{Participantes}

Participaram quatro homens (P2, P4, P5 e P8) e uma mulher (P6), todos universitários, exceto do curso de Psicologia, sem experiência prévia em pesquisas sobre condicionamento operante e sem suspeita ou diagnóstico de Lesão por Esforços Repetitivos (LER).

\section{Local, materiais e instrumentos}

A coleta de dados foi conduzida em um laboratório de uma universidade pública, no qual havia duas salas de aproximadamente $3 \mathrm{~m}^{2}$. Em cada uma das salas havia uma mesa, uma cadeira, um ventilador, um computador do tipo PC (com monitor de 14 polegadas, teclado e mouse padrão). Para isolamento acústico, foi utilizado um gravador com fone de ouvido, para a emissão de um ruído branco (i.e., som constante de um rádio fora da estação). A coleta de dados foi feita com software ProgRef v3.1 (Costa \& Banaco, 2002). A primeira tela do software consistia em um fundo cinza, com apenas um botão e a palavra "Iniciar" na posição central superior da tela. Ao clicar sobre esse botão, a sessão experimental era iniciada com a apresentação de uma segunda tela (Figura 1) que consistia em um fundo cinza, com um retângulo no centro inferior da tela (botão de respostas), um contador de pontos no centro superior da tela e um retângulo no canto superior direito da tela (botão de resposta de consumação). O ProgRef registrava a frequência de respostas e pontos obtidos por minuto, o intervalo entre respostas (IRT) de todas as respostas emitidas na sessão e construía o gráfico de registro cumulativo das respostas de cada sessão.

\section{Procedimento}

Antes do início da primeira sessão, o participante lia e assinava o Termo de Consentimento Livre e Esclarecido (TCLE) ${ }^{1}$ que, em linhas gerais, informava que o objetivo do estudo era "verificar o efeito do custo da resposta sobre o comportamento de pressionar um botão na tela de um computador"; qual o número e a duração das sessões; que deveriam ga-

1 O Experimento 1 foi aprovado pelo Comitê de Ética em Pesquisa Envolvendo Seres Humanos da UEL (Registro CONEP 268); Parecer no 022/05.

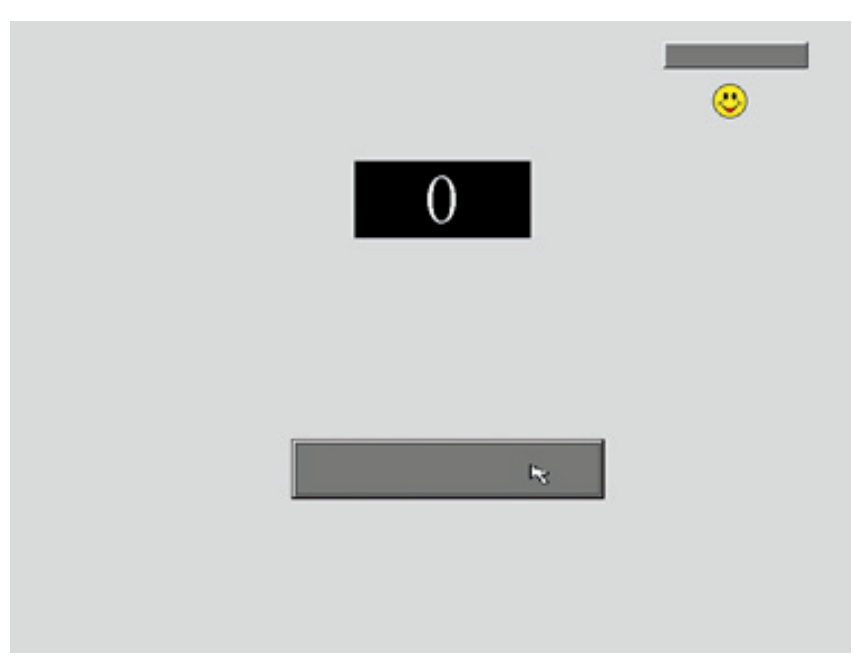

Figura 1. Layout da tela da sessão do ProgRef.

nhar o maior número de pontos usando apenas o mouse; que poderiam deixar a pesquisa a qualquer momento sem qualquer prejuízo; e que receberiam $\mathrm{R} \$ 0,03$ por ponto ao final de cada sessão.

Em seguida, era solicitado ao participante que deixasse todo material, incluindo o relógio e aparelho celular (desligado) em uma mesa do laboratório, fora da sala experimental, e lesse uma folha com as seguintes instruções:

"Obrigado por sua colaboração! Sua tarefa será clicar com o botão esquerdo do mouse sobre um retângulo que aparece na parte inferior do monitor para obter pontos. Abaixo você pode observar o layout da tela. [Uma figura da tela do computador, como a exibida na Figura 1, era apresentada]. Você deve tentar ganhar o maior número de pontos que você conseguir. Você ganhará pontos clicando sobre o botão de uma maneira específica. Os pontos aparecerão na janela que se localiza na parte superior da tela na posição central (visor de pontuação). Entretanto, quando você ganhar algum ponto aparecerá no canto superior direito do monitor um smile. Você deverá, então, clicar com a seta do mouse sobre o botão que se localiza no canto superior direito da tela. Ao fazer isso o smile desaparecerá e o ponto será creditado no contador. Quando um smile aparecer no monitor não aparecerá outro até que você clique no botão menor no canto superior direito. Depois que o smile desaparece - e o ponto é creditado 
- você pode voltar a clicar sobre o botão maior na parte inferior do monitor para ganhar mais pontos. Boa sorte!"

$\mathrm{Na}$ sala experimental, era pedido para que o participante colocasse o fone de ouvido e não o retirasse até o final da sessão. A sessão tinha início assim que o participante clicasse o botão esquerdo do mouse com o cursor sobre o botão escrito "Iniciar".

A tarefa experimental consistia em pressionar o botão esquerdo do mouse com o cursor sobre o botão de respostas. Quando a contingência era cumprida, um smile aparecia no canto superior direito da tela, abaixo do botão de resposta de consumação. O participante deveria, então, clicar com o cursor sobre o botão de resposta de consumação para que o smile desaparecesse e um ponto fosse creditado no contador de pontos. Quando um FI ou um DRL estivesse em vigor, os intervalos desses programas de reforço tinham início logo após o aparecimento do smile e não após a resposta de consumação. Portanto, o tempo necessário para deslocar o cursor do mouse do botão de respostas até o botão de resposta de consumação e clicar sobre ele era computado como parte da pausa pós-reforço. Essa programação da resposta de consumação é semelhante à utilizada em pesquisas com ratos em que água é usada como evento consequente (i.e., uma dada pressão à barra aciona o bebedouro, um novo intervalo é iniciado e o tempo que o rato passa lambendo o bebedouro está dentro do intervalo de um FI ou de um DRL).

A Tabela 1 apresenta um resumo do procedimento experimental. Foram realizadas 12 sessões com a duração de 30 min cada. A cor do botão de resposta era diferente para cada programa de reforço (como em Weiner, 1964, 1969) e os parâmetros dos programas de reforços (FR 60 e FI 15 s) foram idênticos aos utilizados por Costa, Soares et al. (2009).

Fase 1. Os participantes eram expostos a três sessões consecutivas em um programa de reforço de FR 60 no qual a $60^{\text {a }}$ resposta emitida desde o aparecimento do último smile ou do início da sessão era seguida pela apresentação do smile. Durante esta fase, a cor do botão de respostas era cinza.

Fase 2. Todos os participantes eram expostos a três sessões em um programa de reforço em FI
15 s, no qual a primeira resposta emitida após um intervalo de 15 segundos desde o aparecimento do último smile ou desde o início da sessão era seguida pela apresentação do smile. Nesta fase, a cor do botão de respostas era vermelha.

\section{Tabela 1}

Resumo do procedimento do Experimento 1.

\begin{tabular}{lllll}
\hline & \multicolumn{4}{c}{ Fases } \\
\cline { 2 - 5 } & Fase 1 & Fase 2 & Fase 3 & Fase 4 \\
\hline $\begin{array}{l}\text { Programa de } \\
\text { Reforço }\end{array}$ & FR 60 & FI 15 s & FR 60 & DRL 3 s \\
$\begin{array}{l}\text { Cor do Botão } \\
\begin{array}{l}\text { Duração da } \\
\text { Sessão }\end{array}\end{array}$ & Cinza & Vermelho & Cinza & Azul \\
$\begin{array}{l}\text { Número de } \\
\text { sessões }\end{array}$ & 30 min & 30 min & 30 min & 30 min \\
\hline
\end{tabular}

Fase 3. Os participantes eram expostos, por três sessões, ao mesmo programa de reforço da Fase 1 (FR 60). A cor do botão de respostas era cinza.

Fase 4. Nesta fase, que também tinha duração de três sessões, estava em vigor um programa de reforço DRL $3 \mathrm{~s}$, no qual o intervalo entre respostas (IRT) ou o intervalo entre o início da sessão e a primeira resposta deveria ser de, no mínimo, $3 \mathrm{~s}$ para que o smile aparecesse na tela do computador. Caso o participante clicasse no botão de respostas antes de completar o intervalo, o cronômetro zerava e reiniciava a contagem do tempo. A cor do botão de respostas nesta fase era azul.

\section{Resultados}

A Figura 2 apresenta, para cada participante, a taxa de respostas (R/min) em cada sessão (círculos brancos) e a taxa média de respostas em cada fase (linhas horizontais pontilhadas). As linhas tracejadas verticais indicam a mudança de fases do experimento. Os números nas fases de FR indicam a quantidade total de pontos (reforços) obtidos nas respectivas fases.

Ao longo da primeira exposição ao FR, todos os participantes emitiram taxas de respostas relativamente altas (entre 108 e $344 \mathrm{R} / \mathrm{min}$ ). Quando a contingência mudou de FR para FI, três dos cinco participantes (P2, P5 e P6) mantiveram taxas de respostas semelhantes às da fase anterior (acima de $132 \mathrm{R} /$ min na última sessão de FR e acima de 123 


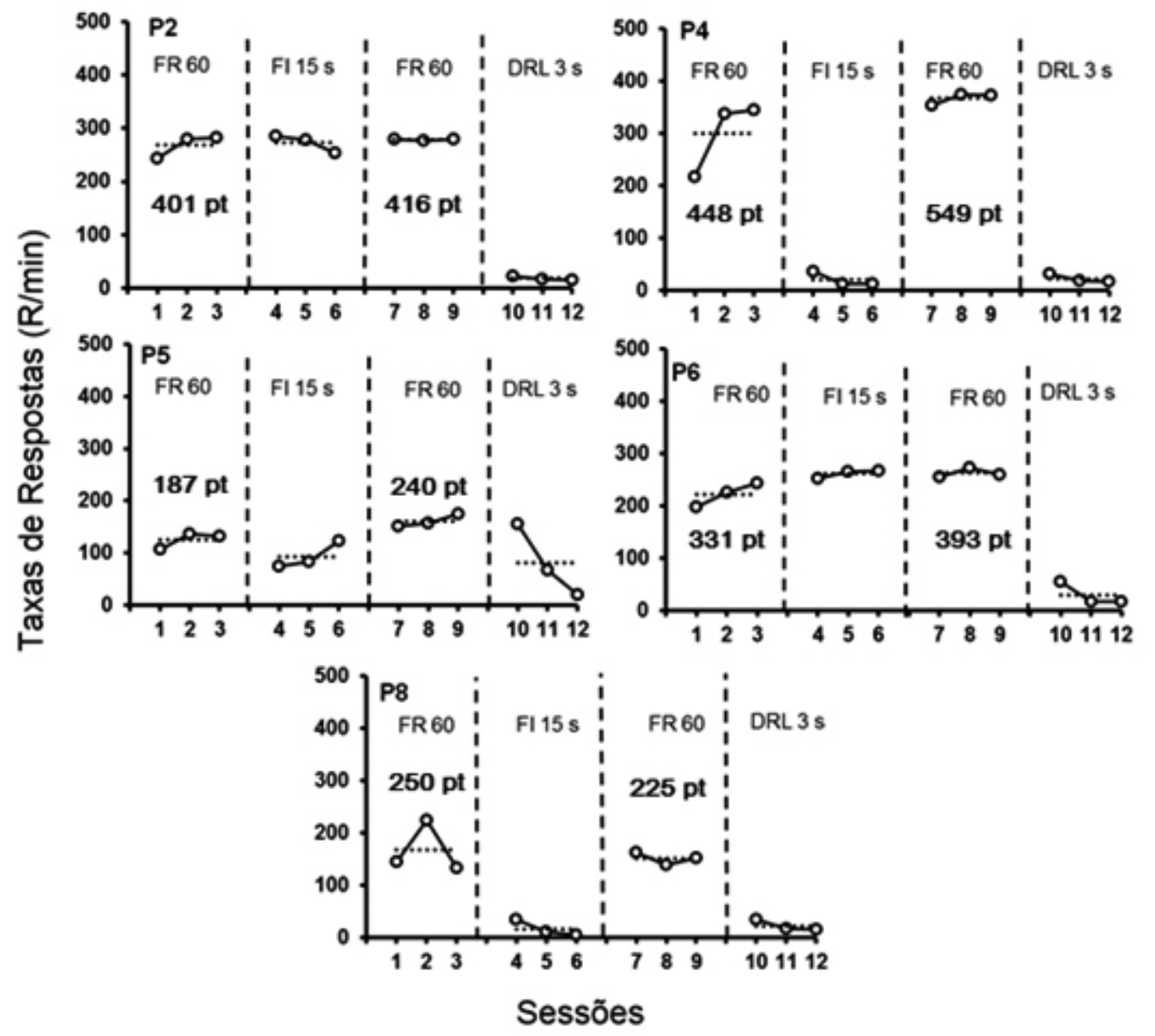

Figura 2. Taxa de respostas $(\mathrm{R} / \mathrm{min})$ em cada sessão para cada participante (linhas contínuas cujos marcadores são círculos brancos). As linhas pontilhadas horizontais representam a média das taxas de respostas em cada fase. Os números nas fases de FR indicam a quantidade total de pontos (reforços) obtidos nas respectivas fases.

$\mathrm{R} /$ min na última sessão de FI). Os outros dois participantes diminuíram a taxa de respostas quando a contingência mudou do FR para o FI (344 e 133 $\mathrm{R} /$ min na última sessão de exposição ao FR e 12 e 5 $\mathrm{R} /$ min na última sessão de exposição ao FI, para $\mathrm{P} 4$ e $\mathrm{P} 8$ respectivamente).

Durante a segunda exposição ao FR (Fase 3), todos os participantes emitiram taxas de respostas relativamente altas (acima de $139 \mathrm{R} / \mathrm{min}$ ). Quando a contingência mudou de FR para DRL (Fase 4), a taxa de respostas de todos os participantes tendeu a diminuir, sendo que, para P2, P4, P6 e P8, a redução ocorreu na primeira sessão de exposição ao DRL (taxa de respostas abaixo de $54 \mathrm{R} / \mathrm{min}$ ). Para $\mathrm{P} 5$, na primeira sessão de exposição ao DRL, a taxa de respostas permaneceu semelhante à da fase anterior $(156 \mathrm{R} / \mathrm{min})$, porém, na segunda sessão de
DRL, ocorreu uma redução na taxa de respostas. $\mathrm{Na}$ última sessão de exposição ao DRL, todos os participantes emitiram taxas de respostas abaixo de $19 \mathrm{R} / \mathrm{min}$. Todos os participantes, exceto P8, marcaram mais pontos na Fase 1 do que na Fase 3.

A Figura 3 apresenta o registro cumulativo dos últimos 5 minutos de exposição ao FR (Fase 1 e Fase 2) e dos primeiros 15 minutos de exposição ao FI e ao DRL.

Observa-se, na Figura 3, que todos os participantes emitiram taxas de respostas relativamente altas e constantes nos últimos 5 minutos de exposição ao FR (acima de $150 \mathrm{R} / \mathrm{min}$ neste período) nas Fases 1 e 3. Ao longo dos primeiros 15 minutos de exposição ao FI, P2 e P6 mantiveram taxas de respostas relativamente altas e constantes. O P5 emitiu, desde o início da exposição ao FI, taxas de respostas relati- 


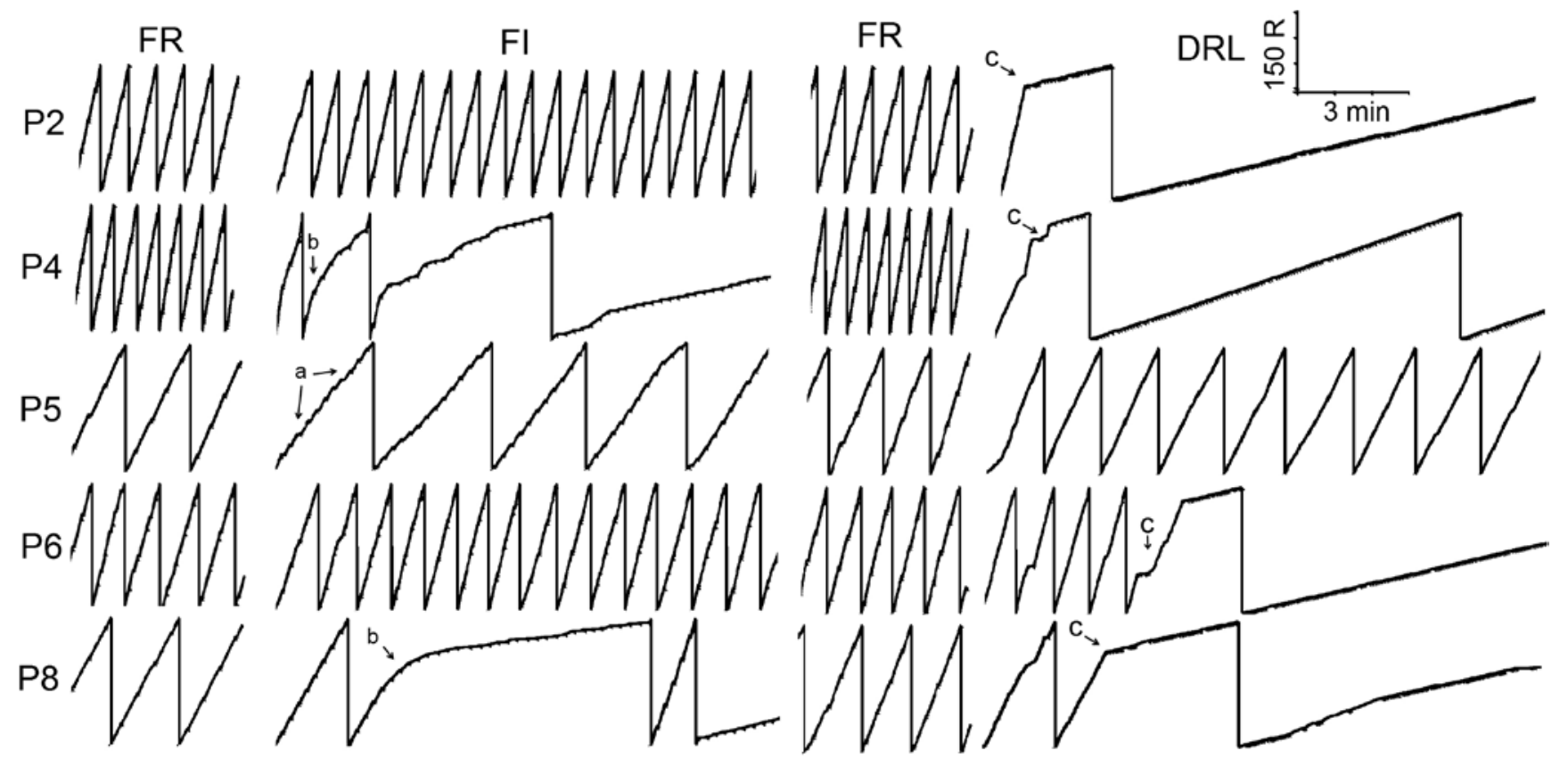

Figura 3. Frequência acumulada de respostas de cada participante dos últimos 5 minutos de exposição ao FR (Fase $1-1^{\text {a }}$ coluna e Fase 3 - 3a coluna) e dos 15 primeiros minutos de exposição ao FI (Fase $2-2^{\text {a }}$ Coluna) e ao DRL (Fase $4-4^{\text {a }}$ Coluna). O registro cumulativo da taxa de respostas voltava a zero após 250 respostas. As marcas diagonais na curva da taxa de respostas indicam o aparecimento do smile.

vamente menores que a da última sessão de FR (ver também Figura 2), com um padrão de distribuição das respostas que se assemelha a um break-and-run (exemplificado na Figura 3 pelas setas com a letra $a$ ); entretanto, como observado na Figura 2, sessões 5 e 6 , a taxa de respostas aumentou ao longo da exposição ao FI para esse participante. P4 e P8 emitiram, inicialmente, taxas de respostas semelhantes àquelas observadas durante o FR, porém, diminuíram a taxa de respostas até o quarto minuto de exposição ao FI (a seta com a letra $b$ exibe o momento em que a taxa de respostas em FI começa a se alterar).

Quando a contingência mudou de FR para DRL, todos os participantes emitiram taxas de respostas relativamente altas no início do DRL (até, pelo menos, o primeiro minuto - quarta coluna da Figura 3) e, ao longo da exposição ao DRL, a taxa de respostas diminuiu (setas com a letra $c$ ). Apenas P5 manteve taxas de respostas relativamente altas ao longo da primeira sessão de DRL, sendo que, na segunda sessão de exposição a esse programa de reforço, a taxa de respostas diminuiu (ver Figura 2).
A Tabela 2 exibe o tempo (em minutos e segundos) para a obtenção do primeiro smile (reforço) e a porcentagem de pontos obtidos, relativo ao total de pontos disponíveis, para a primeira sessão da Fase 2 (FI) e para a primeira e última sessão da Fase 4 (DRL). Para o P5, os dados do DRL se referem a segunda sessão, pois este participante não obteve nenhum smile durante os 30 minutos da primeira sessão.

Observa-se na Tabela 2 que todos os participantes, na primeira sessão de FI, obtiveram o primeiro smile aos 15 segundos de sessão (exatamente o parâmetro temporal do FI) e obtiveram mais de $90 \%$ dos pontos disponíveis na sessão. Na primeira sessão do DRL $3 \mathrm{~s}$, a maioria dos participantes obteve o primeiro smile após 50 segundos de sessão. P5 não obteve smile na primeira sessão, vindo a obtê-lo apenas após transcorridos mais de 7 minutos da segunda sessão de DRL. Embora P8 tenha obtido o primeiro smile após 4 segundos da primeira sessão, é possível sugerir que a obtenção desse smile tenha sido "acidental" (i.e., o participante demorou 


\section{Tabela 2. \\ Tempo Para Obtenção Do Primeiro Smile e a Porcentagem De Pontos Obtidos Na Primeira Sessão De FI (Fase 2) e Na Primeira e Última Sessão De DRL (Fase 4) Dos Participantes Do Experimento 1.}

\begin{tabular}{|c|c|c|c|c|c|c|}
\hline \multirow[b]{3}{*}{ Participantes } & \multirow{2}{*}{\multicolumn{2}{|c|}{$\begin{array}{c}\text { Fase } 2 \text { - FI } \\
\text { Primeira Sessão }\end{array}$}} & \multicolumn{4}{|c|}{ Fase 4 - DRL } \\
\hline & & & \multicolumn{2}{|c|}{ Primeira Sessão } & \multicolumn{2}{|c|}{ Última Sessão } \\
\hline & $\begin{array}{l}\text { Tempo } \\
\text { (mm:ss) }\end{array}$ & Porcent. & $\begin{array}{l}\text { Tempo } \\
\text { (mm:ss) }\end{array}$ & Porcent. & $\begin{array}{l}\text { Tempo } \\
\text { (mm:ss) }\end{array}$ & Porcent. \\
\hline $\mathrm{P} 2$ & $00: 15$ & 99 & $00: 50$ & 77 & $00: 12$ & 75 \\
\hline P4 & $00: 15$ & 96 & $01: 11$ & 53 & 00:07 & 77 \\
\hline P5 & $00: 15$ & 97 & $07: 27^{\star}$ & $47^{*}$ & 00:06 & 67 \\
\hline P6 & $00: 15$ & 98 & $01: 25$ & 65 & 00:07 & 81 \\
\hline P8 & $00: 15$ & 92 & 00:04 & 37 & $00: 10$ & 63 \\
\hline
\end{tabular}

Nota: mm:ss = minutos:segundos; Porcent. $=$ Porcentagem .

* Dados da segunda sessão de DRL.

4 segundos entre clicar no botão de iniciar a sessão e dar a primeira pressão no botão de respostas). P8 levou 1 min $39 \mathrm{~s}$ e 1 min $32 \mathrm{~s}$ para obter o segundo e terceiro smile, respectivamente (dados não exibidos na tabela). Todos os participantes obtiveram $77 \%$ ou menos dos pontos disponíveis na primeira sessão do DRL (P5 obteve $47 \%$ dos pontos na segunda sessão do DRL e nenhum na primeira sessão). Na última sessão do DRL, todos os participantes, exceto P8, diminuíram o tempo para a obtenção do primeiro smile e aumentaram, exceto P2, a porcentagem de pontos obtidos em relação a primeira sessão do DRL (63\% ou mais, dos pontos disponíveis).

\section{Discussão}

As Fases 1 e 2 do presente estudo foram uma replicação sistemática da pesquisa de Costa et al. (2008) e replicaram parcialmente aqueles resultados. No estudo de Costa et al., todos os participantes submetidos a FR 40 e, depois, FI $10 \mathrm{~s}$ mantiveram altas taxas de respostas sob o FI quando pontos eram trocados por dinheiro ou fotocópias. No presente estudo, três de cinco participantes mantiveram taxas de respostas relativamente altas. As principais diferenças entre os estudos foram a taxa de reforço em ambas as fases, a magnitude do reforço e a duração da sessão (FR 40 e FI 10 s; R $\$ 0,05$ por ponto; e 15 min no estudo de Costa et al., 2008 vs. FR 60 e FI 15 s; R $\$ 0,03$ por ponto; e 30 min no presente estudo). Apesar destas diferenças de procedimento, a manutenção da taxa de respostas durante as três sessões de FI foi observada para a maioria dos participantes (três de cinco).

Quando a contingência de reforço mudou de FR para DRL (Fases 3 e 4), a taxa de respostas diminuiu para todos os cinco participantes. Esses resultados foram obtidos em outras pesquisas nas quais, pelo menos parte das manipulações experimentais, envolviam a transição de um FR para um DRL (e.g., Cole, 2001; Wanchisen et al., 1998; Weiner, 1969). A diferença fundamental da transição do FR para FI e do FR para DRL é que o FI permite ampla variação na taxa de respostas sem afetar a taxa de reforço o que não ocorre com o DRL, sugerindo ser precipitada a interpretação da persistência de altas taxas de respostas, a despeito da mudança da contingência de reforço, como um caso de "insensibilidade comportamental" (cf. Hayes, Brownstein, Zettle, et al., 1986; Hayes, Brownstein, Haas et al., 1986; Rosenfarb, et al., 1992; Wulfert et al., 1994).

Em certa medida, a transição de FR para DRL pode ser comparada à transição de FR para FIcusto (e.g., Costa et al., 2013; Costa, Soares et al., 2009): em ambos os casos, a manutenção de altas taxas de respostas implicou na diminuição de reforçadores (na obtenção de menos pontos em FIcusto ou nenhum ponto em DRL). Costa, Cirino et al. (2009) apontaram que "Os efeitos [da história comportamental] durarão enquanto as contingências [atuais] o permitirem" (p. 401). O resultado do Experimento 1 sugere que o efeito da história é determinado, pelo menos em parte, pela contingên- 
cia atual (cf. LeFrancois \& Metzger, 1993; Weiner, 1983), isto é, a persistência comportamental de um padrão selecionado na história de reforço é observada quando a contingência atual permite a manutenção de reforçadores (como ocorreu na alteração da contingência de FR para FI no presente estudo).

Embora estudos sobre momentum comportamental sugiram que a taxa de reforço pode aumentar a resistência do comportamento a mudança (e.g., dos Santos, 2005; Nevin, Mandell, \& Atak, 1983), os resultados deste estudo apontaram que a quantidade total de pontos obtidos em FR foi menor na Fase 1 do que na Fase 3 e a taxa de respostas tendeu a ser menos resistente a mudança sob DRL (Fase 4) do que sob FI (Fase 3). Todavia, em um conjunto de experimentos, Cohen et al. (1993) - assim como no presente experimento - não demonstraram relação consistente entre a resistência do comportamento a mudanças e a taxa de reforço em programas de reforço simples, embora tenham encontrado tal relação em programas de reforço múltiplos.

Apesar da mudança nas cores do botão de respostas correlacionado com cada contingência de reforço ter sido adotada, seguindo os procedimentos básicos de outros estudos (e.g., Costa et al., 2008; Costa, Soares et al., 2009; Weiner, 1964, 1969), não é possível separar completamente o efeito da relação resposta-reforçador - diferente no FI e no DRL - do efeito da mudança na cor do botão de respostas. Resta, assim, ao menos uma questão relevante: qual foi o efeito da mudança na cor do botão de respostas, correlacionada com a mudança na contingência de reforço, sobre a probabilidade de mudança nas taxas de respostas observadas no Experimento 1? O Experimento 2 procurou investigar essa questão.

\section{Experimento 2}

O Experimento $2^{2}$ teve por objetivo investigar o efeito sobre a taxas de respostas selecionadas pela exposição a FR, quando a contingência de reforço foi alterada para uma contingência pouco restriti-

2. O Experimento 2 foi aprovado pelo Comitê de Ética em Pesquisa Envolvendo Seres Humanos da UEL (Registro CONEP 268); Parecer $n^{\circ}$ 190/07. va (FI) e uma contingência muito restritiva (DRL), como realizado no Experimento I, agora sem mudança na cor do botão de respostas correlacionada com cada contingência de reforço.

\section{Método}

\section{Participantes}

Participaram três homens (P9, P12 e P13) e uma mulher (P10), todos universitários. Os critérios para o recrutamento de participantes para a pesquisa foram os mesmos do Experimento I.

\section{Local, materiais e instrumentos}

O local, os materiais e instrumentos foram os mesmos utilizados no Experimento I.

\section{Procedimento}

O procedimento foi similar ao utilizado no Experimento I, diferindo apenas em relação ao número de sessões e à cor do botão de respostas. A Tabela 3 resume as fases do procedimento experimental.

\section{Tabela 3. \\ Resumo do procedimento do Experimento 2.}

\begin{tabular}{lllll}
\hline & \multicolumn{4}{c}{ Fases } \\
\cline { 2 - 5 } & Fase 1 & Fase 2 & Fase 3 & Fase 4 \\
\hline Programa de & FR 60 & FI 15 s & FR 60 & DRL 3 s \\
Reforço & Cinza & Cinza & Cinza & Cinza \\
Cor do Botão & 30 min & 30 min & 30 min & 30 min \\
Duração de Sessão & 30 & 6 & 6 \\
Número de sessões & 6 & 6 & 6 & 6
\end{tabular}

Em todas as fases, a cor do botão era cinza. Os quatro participantes foram expostos a seis sessões em cada fase (em vez de três sessões, como no Experimento 1) com o intuito de diminuir a variabilidade nas taxas de respostas entre as sessões, como ocorreu no Experimento 1. Durante as Fases 1 e 3 estava em vigor um programa FR 60, durante a Fase 2 um FI 15 s e durante a Fase 4 estava em vigor um DRL $3 \mathrm{~s}$. Ao final de cada sessão os participantes receberam $\mathrm{R} \$ 0,03$ para cada ponto obtido. 


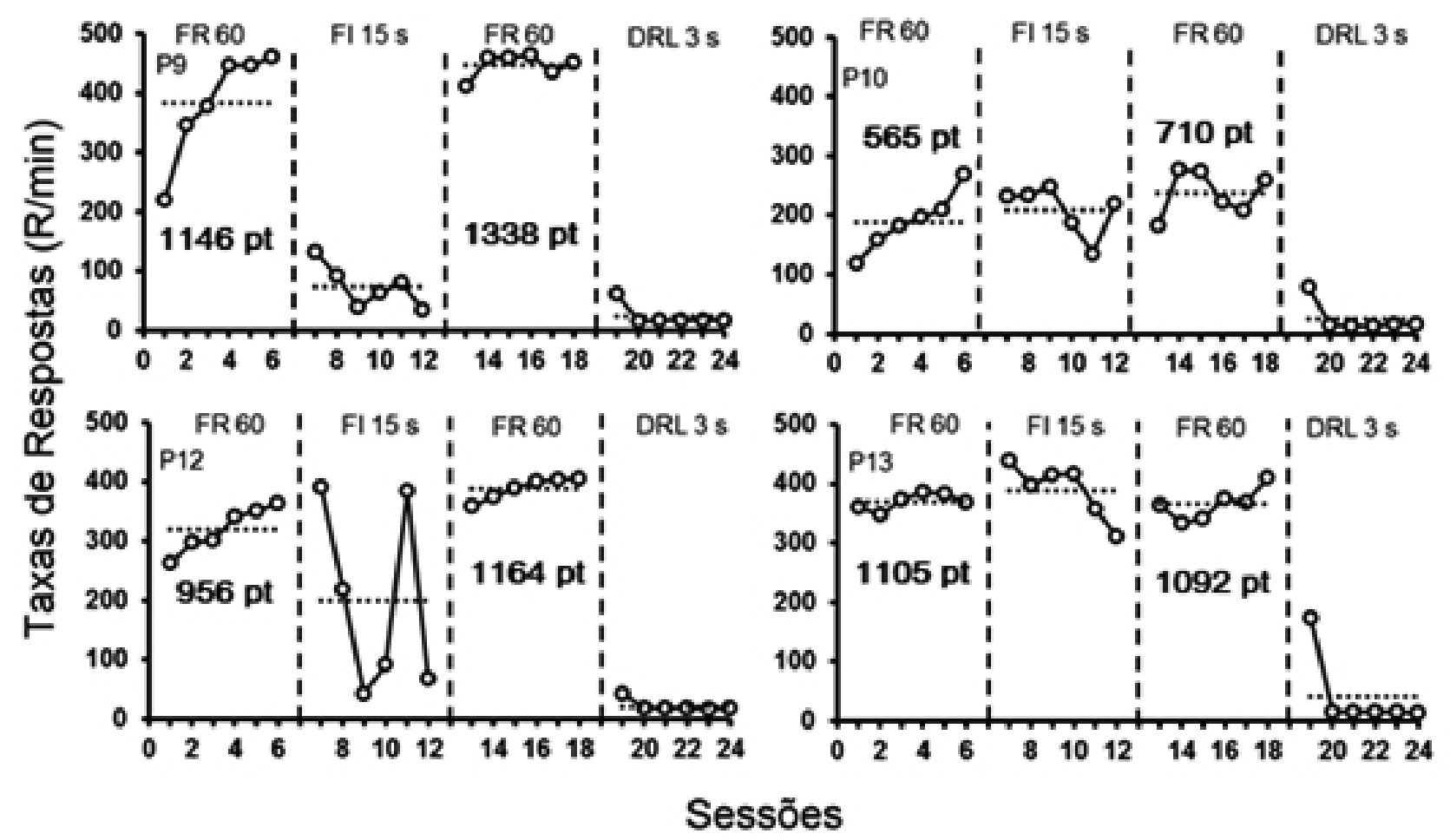

Figura 4. Taxa de respostas (R/min) em cada sessão para cada participante (linhas contínuas cujos marcadores são círculos brancos). As linhas pontilhadas horizontais representam a média das taxas de respostas em cada fase. Os números nas fases de FR indicam a quantidade total de pontos (reforços) obtidos nas respectivas fases.

\section{Resultados}

A Figura 4 apresenta, para cada participante, a taxa de respostas $(\mathrm{R} / \mathrm{min})$ em cada sessão (círculos brancos) e a taxa média de respostas em cada fase (linhas horizontais pontilhadas). As linhas tracejadas verticais indicam a mudança de fases do experimento. Os números nas fases de FR indicam a quantidade total de pontos (reforços) obtidos nas respectivas fases.

Observa-se que, ao longo exposição ao FR (Fase 1), os participantes P9, P10 e P12 apresentaram uma tendência de aumento na taxa de respostas. $\mathrm{Na}$ última sessão de exposição ao FR (Fase 1), a taxa de respostas para todos os quatro participantes foi acima de $269 \mathrm{R} / \mathrm{min}$ (média acima de $188 \mathrm{R} / \mathrm{min}$, linhas pontilhadas). Quando a contingência mudou de FR para FI (Fase 2), dois dos quatro participantes (P10 e P13) mantiveram a taxa de respostas semelhantes àquelas da última sessão de FR (média acima de $199 \mathrm{R} / \mathrm{min}$, linhas pontilhadas). Apesar disso, é possível notar uma tendência das taxas de respostas diminuírem ao longo da exposição continuada ao FI, pelo menos para P9, P10 e P13. O participante P12 variou as taxas de respostas entre sessões, sendo que em duas delas (Sessões 7 e 11), a taxa foi semelhante à da Fase $1 \mathrm{e}$, nas demais, abaixo das emitidas na fase anterior; na média, a taxa de respostas diminuiu da Fase 1 para a Fase 2 (i.e., média de 319 R/min na Fase 1 e 199 R/min na Fase 2, linhas pontilhadas). P9 diminuiu a taxa de respostas desde a primeira sessão de exposição ao FI (passou de $461 \mathrm{R} / \mathrm{min}$ na última sessão de FR, para $132 \mathrm{R} /$ min na primeira sessão de FI).

Durante a segunda exposição ao FR (Fase 3), todos os participantes voltaram a emitir altas taxas de respostas (acima de $183 \mathrm{R} / \mathrm{min}$ e média superior a $237 \mathrm{R} / \mathrm{min}$, linhas pontilhadas). Quando a contingência mudou de FR para DRL, a taxa de respostas de todos os participantes diminuiu desde a primeira sessão de exposição a este programa de reforço (taxa de respostas abaixo de $173 \mathrm{R} / \mathrm{min}$ na primeira sessão do DRL). Na última sessão de exposição 

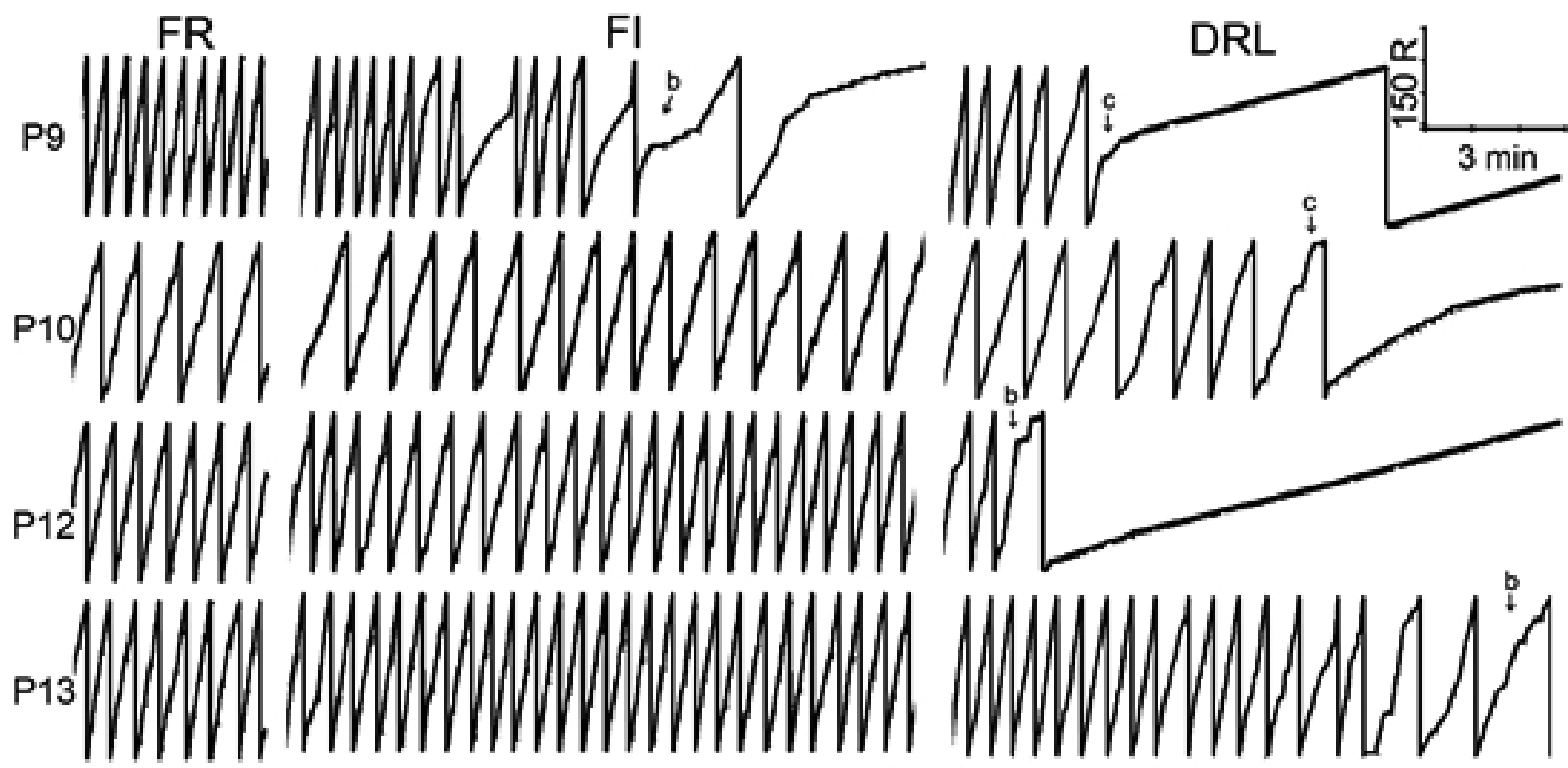

Figura 5. Frequência acumulada de respostas de cada participante dos últimos cinco minutos de exposição ao FR 60 (Fase $1-1^{\text {a }}$ coluna) e dos 15 primeiros minutos de exposição ao FI (Fase $2-2^{\text {a }}$ Coluna) e ao DRL (Fase $4-3^{a}$ Coluna). O desempenho exibido em FR durante a Fase 1 é representativo do desempenho durante a Fase 3 (não mostrado na figura). A curva da taxa de resposta volta a zero após ocorrência de 250 respostas. As marcas diagonais na curva da taxa de resposta indicam o aparecimento do smile.

ao DRL, todos os participantes emitiram taxa de respostas menores que $18 \mathrm{R} / \mathrm{min}$. Os participantes marcaram mais pontos na Fase 1 do que na Fase 3, exceto P13.

A Figura 5 apresenta o registro cumulativo dos últimos cinco minutos de exposição ao FR (Fase 1) e dos primeiros quinze minutos de exposição ao FI e DRL.

Observa-se, na Figura 5, que todos os participantes apresentaram taxa de respostas relativamente alta nos últimos 5 minutos de exposições ao FR (acima de $300 \mathrm{R} / \mathrm{min}$ ). Ao longo dos 15 minutos da primeira sessão de exposição ao FI, três dos quatro participantes (P10, P12 e P13) permaneceram com um padrão de respostas semelhante ao observado durante a exposição ao FR. P9 apresentou, inicialmente, um padrão de respostas semelhante ao apresentado durante o FR, porém, diminuiu a taxa de respostas ao longo da exposição ao FI (a seta com a letra $b$ exibe o momento em que a taxa de respostas começa a alterar).

O desempenho dos participantes na Fase 1 (primeira coluna da Figura 5) é representativo do desempenho nos 5 minutos finais da exposição ao FR na Fase 3. Quando a contingência mudou de FR para DRL, observa-se, na Figura 5, que também houve taxas de respostas relativamente altas durante, pelo menos, os dois primeiros minutos de exposição ao DRL para todos os participantes, de forma bastante similar à taxa de respostas emitida durante a exposição ao FR. Ao longo da exposição ao DRL a taxa de respostas diminuiu (setas marcadas pela letra $c$ ), para todos os participantes.

A Tabela 4 exibe o tempo (em minutos e segundos) para a obtenção do primeiro smile (reforço) e a porcentagem de pontos obtidos, relativo ao total de pontos disponíveis, para a primeira sessão da Fase 2 (FI) e para a primeira e última sessão da Fase 4 (DRL).

Assim como no Experimento 1, observa-se na Tabela 4 que todos os participantes do Experimento 2, na primeira sessão de FI, obtiveram o primeiro smile aos 15 segundos de sessão (exatamente o parâmetro temporal do FI) e obtiveram $97 \%$ ou mais dos pontos disponíveis na sessão. Na primeira sessão do DRL, todos os participantes obtiveram o primeiro smile apenas depois de transcorridos mais 


\section{Tabela 4. \\ Tempo Para Obtenção Do Primeiro Smile e a Porcentagem De Pontos Obtidos Na Primeira Sessão De Fl (Fase 2) e Na Primeira e Última Sessão De DRL (Fase 4) Dos Participantes Do Experimento 2.}

\begin{tabular}{|c|c|c|c|c|c|c|}
\hline \multirow[b]{3}{*}{ Participantes } & \multirow{2}{*}{\multicolumn{2}{|c|}{$\begin{array}{c}\text { Fase } 2 \text { - FI } \\
\text { Primeira Sessão }\end{array}$}} & \multicolumn{4}{|c|}{ Fase 4 - DRL } \\
\hline & & & \multicolumn{2}{|c|}{ Primeira Sessão } & \multicolumn{2}{|c|}{ Última Sessão } \\
\hline & $\begin{array}{l}\text { Tempo } \\
\text { (mm:ss) }\end{array}$ & Porcent. & $\begin{array}{l}\text { Tempo } \\
\text { (mm:ss) }\end{array}$ & Porcent. & $\begin{array}{l}\text { Tempo } \\
\text { (mm:ss) }\end{array}$ & Porcent. \\
\hline P9 & $00: 15$ & 97 & $03: 54$ & 68 & $00: 07$ & 84 \\
\hline P10 & $00: 15$ & 98 & $05: 22$ & 40 & 00:07 & 73 \\
\hline P12 & $00: 15$ & 99 & $01: 56$ & 72 & $00: 08$ & 81 \\
\hline P13 & $00: 15$ & 99 & $10: 51$ & 27 & $00: 05$ & 69 \\
\hline
\end{tabular}

Nota: $\mathrm{mm}: \mathrm{ss}=$ minutos:segundos; Porcent. $=$ Porcentagem .

de 1 min e 55 s de sessão. P13 obteve o primeiro smile apenas 10 min e $51 \mathrm{~s}$ após iniciada a primeira sessão. Todos os participantes obtiveram $72 \%$ ou menos do total de pontos disponíveis na primeira sessão do DRL. Na última sessão do DRL, todos os participantes diminuíram o tempo para a obtenção do primeiro smile (até $8 \mathrm{~s}$ ) e aumentaram a porcentagem de pontos obtidos em relação a primeira sessão do DRL (acima de 69\%).

\section{Discussão}

No Experimento 2 houve, assim como no Experimento 1, variabilidade entre os participantes em relação à transição da Fase 1 (FR) para a Fase 2 (FI). Dois dos quatro participantes mantiveram altas taxas de respostas no FI (P10 e P13); um participante diminuiu a taxa de respostas (P9) - apesar de ter mantido altas taxas de respostas no primeiro quarto da primeira sessão (ver Figura 5) - e um participante variou a taxa de respostas entre as sessões de FI (P13). Esses resultados gerais indicam que a manutenção da cor do botão de respostas entre as Fases 1 e 2, não aumentou a probabilidade de que as altas taxas de respostas selecionadas no FR se mantivessem no FI. Variabilidade na taxa de respostas entre e intra participantes expostos ao FI têm sido relatadas em diversos estudos (e.g., Costa, Banaco, \& Becker, 2005; Costa, Patsko, \& Becker, 2007; Okouchi, 2002; Weiner, 1969). Apesar desse resultado geral, uma comparação entre os registros cumulativos da primeira sessão de FI, após exposição ao FR, sugere que a taxa de respostas tendeu a demorar um pouco mais para mudar no Experimento 2 (sem mudança de estímulos) do que no Experimento 1 (com mudança na cor do botão de respostas).

No Experimento 2, o estímulo correlacionado com as contingências de reforço foram retiradas, mas também se aumentou o número de sessões de exposição a cada programa de reforço, o que pode ter favorecido a maior persistência, dado que o padrão de respostas foi reforçado por mais tempo (cf. dos Santos, 2005; Nevin, Mandell, \& Atak, 1983; e a teoria do momentum comportamental). Todavia, como apontado anteriormente, em programas simples de reforço, a taxa (ou quantidade) de reforço não prediz a resistência do comportamento a mudança (Cohen et al., 1983) e, portanto, a diferença encontrada na primeira sessão de FI entre o Experimento 1 e 2 talvez não seja explicada simplesmente pela diferença na qualidade de reforço. De qualquer modo, uma replicação do presente estudo que controlasse a taxa e a quantidade de reforço entre os dois procedimentos poderia lançar mais luz sobre essa questão.

A despeito dessa variabilidade durante a exposição ao FI, quando a contingência retornou para FR (Fase 3), todos os participantes voltaram (ou continuaram) a emitir altas taxas de respostas. Quando a contingência mudou de FR para DRL, todos os quatro participantes diminuíram a taxa de respostas logo na primeira sessão do DRL (ver Figura 5), indicando que o desempenho dos participantes P10 e P13 não era, necessariamente, in- 
sensíveis às contingências de reforço na transição do FR para o FI, i.e., quando a manutenção do desempenho selecionado na história implicou na não obtenção de pontos, o desempenho mudou.

\section{Discussão geral}

De modo geral, observou-se que (a) quando o programa de reforço foi alterado, mas pontos continuaram disponíveis mesmo que o participante variasse a taxa de respostas para mais ou para menos (FI), a taxa de respostas tendeu a diminuir para quatro dos nove participantes; (b) quando a contingência mudou e os pontos deixaram de ser obtidos (DRL), o comportamento tendeu a alterar-se de altas para baixas taxas de respostas, o que garantiu a obtenção de pontos, para todos os participantes. Esse resultado geral não dependeu da mudança de estímulos entre as diversas fases (Experimento 1 vs. Experimento 2), exceto, talvez, se considerados os minutos iniciais de exposição ao FI (ver Figuras 3 e 5).

Esses resultados sugerem ser precipitada uma análise da manutenção de altas taxas de respostas em um programa de FI como um caso de insensibilidade do comportamento às contingências (cf. Bentall \& Lowe, 1987; Bentall, Lowe, \& Beasty, 1985; Lowe, 1979; Lowe, Beasty, \& Bentall, 1983). A alteração do comportamento requer uma relação funcional entre a resposta (ou uma sequência de respostas) e a consequência produzida. Supor que o comportamento deva mudar porque a relação entre resposta-reforçador foi estruturalmente alterada (pela manipulação do experimentador), a despeito do comportamento do organismo poder fazer contato com esta mudança, é uma análise mais estrutural do que funcional da relação organismo-ambiente.

Experimentos sobre a sensibilidade do comportamento governado por regras em contraposição ao modelado por contingências (Skinner, 1969, 1974) tem sugerido que o controle instrucional tende a ser abandonado quando as instruções levam a perda de reforço, seja pela perda de reforçadores ou a não obtenção de reforçadores (Buskist \& Miller Jr., 1986; Galizio, 1979; Paracampo \& Albuquerque, 2004). Paracampo e Albuquerque sugeriram que o abandono do seguimento de regras pode depender do contato com as consequências diferenciais para o seguimento e não seguimento das regras (e.g., não obtenção de reforço para o seguimento da regra e obtenção de reforço por não segui-la). O presente estudo, embora não envolva diretamente o controle instrucional, mas o efeito da exposição prévia a um programa de reforço em FR, aponta para uma conclusão semelhante: enquanto o padrão comportamental selecionado na história de reforço ainda produz reforçadores (FI), o efeito da história persiste sobre o comportamento atual, mas se a manutenção do padrão selecionado na história não garantir mais o ganho de pontos e pontos forem obtidos pela emissão de um padrão diferente de respostas (DRL), o comportamento selecionado não persistirá (ver dos Santos, 2005, para um discussão sobre a relação entre os temas de pesquisa momentum comportamental, história comportamental e comportamento governado por regras).

Alguns estudos sobre custo da resposta em FI com humanos têm apontado para resultados semelhantes aos do presente estudo, isto é, quando a manutenção de altas taxas de respostas implica na perda de reforçadores, há maior probabilidade na redução nas taxas de respostas, após história de FR, em relação a uma condição em que a manutenção das altas taxas de respostas não implica perda de reforçadores (Costa et al., 2013; Costa, Soares et al., 2009).

\section{Referências}

Albuquerque, L.C. (2001). Definições de regras. In H. J. Guilhardi, M. B. B. P. Madi, P. B. P. S. Queiroz \& M. C. Scoz (Eds.), Sobre comportamento e cognição: Expondo a variabilidade (Vol. 7, pp. 132-140). Santo André, SP: ESETec.

Aló, R.M. (2005). História de reforçamento. In J. Abreu-Rodrigues \& M. R. Ribeiro (Eds.), Análise do comportamento: Pesquisa, teoria e aplicação (pp. 45-62). Porto Alegre, RS: Artmed.

Bentall, R.P., \& Lowe, C.F. (1987). The role of verbal behavior in human learning: III. Instructional effects in children. Journal of the Experimental Analysis of Behavior, 47(2), 177-190.

Bentall, R.P., Lowe, C.F., \& Beasty, A. (1985). The role of verbal behavior in human learning: II. Developmental differences. Journal of the Experimental Analysis of Behavior, 43(2), 165-180. 
Buskist, W.F., \& Miller JR., H.L. (1986). Interaction between rules and contingencies in the control of human fixed-interval performance. Psychological Record, 36(1), 109-116.

Cohen, S.L., Riley, D.S., \& Weigle, P.A. (1993). Tests of behavior momentum in simple and multiple schedules with rats and pigeons. Journal of the Experimental Analysis of Behavior, 60(2), 255291.

Cole, M.R. (2001). The long-term effect of high- and low-rate responding histories on fixed-interval responding in rats. Journal of the Experimental Analysis of Behavior, 75(1), 43-54.

Costa, C.E., \& Banaco, R.A. (2002). Progref v3: Sistema computadorizado para coleta de dados sobre programas de reforço com humanos - recursos básicos. Revista Brasileira de Terapia Comportamental e Cognitiva, 4(2), 173-192.

Costa, C.E., Banaco, R.A., \& Becker, R.M. (2005). Desempenho em FI com humanos: Efeito do tipo de reforçador. Temas em Psicologia da SBP, 13(1), 18-33.

Costa, C.E., Banaco, R.A., Longarezi, D.M., Martins, E.V., Maciel, E.M., \& Sudo, C.H. (2008). Tipo de reforçador como variável moduladora dos efeitos da história em humanos. Psicologia: Teoria e Pesquisa, 24(2), 251-262.

Costa, C.E., Cirino, S.D., Cançado, C.R.X., \& Soares, P.G. (2009). Polêmicas sobre história comportamental: Identificação de seus efeitos e sua duração. Psicologia: Reflexão e Crítica, 22(3), 394-403.

Costa, C.E., de Souza, J.P.P., Bianchini, T., Porto, T.H., \& de Freitas, L.A.B. (2013). Diferentes magnitudes do custo da resposta em um múltiplo FI FI, após uma história em um múltiplo FR FR. Interação em Psicologia, 17(1), 11-26.

Costa, C.E., Patsko, C.H., \& Becker, R.M. (2007). Desempenho em FI com humanos: Efeito da interação da resposta de consumação e do tipo de instrução. Interação em Psicologia, 11(2), 175-186.

Costa, C.E., Soares, P.G., Becker, R.M., \& Banaco, R.A. (2009). O efeito da magnitude do custo da resposta e do evento consequente empregado sobre o comportamento em FI após uma história de FR. Revista Brasileira de Análise do Comportamento, 5(2), 89-106. dos Santos, C.V. (2005). Momento comportamental. In J. Abreu-Rodrigues \& M. R. Ribeiro (Eds.), Análise do comportamento: Pesquisa, teoria e aplicação (pp. 63-80). Porto Alegre, RS: Artmed.

Galizio, M. (1979). Contingency-shaped and rule-governed behavior: Instructional control of human loss avoidance. Journal of the Experimental Analysis of Behavior, 31(1), 53-70.

Hayes, S.C., Brownstein, A.J., Haas, J.R., \& Greenway, D.E. (1986). Instructions, multiple schedules, and extinction: Distinguishing rule-governed from schedule-controlled behavior. Journal of the Experimental Analysis of Behavior, 46(2), 137-147.

Hayes, S.C., Brownstein, A.J., Zettle, R.D., Rosenfarb, I., \& Korn, Z. (1986). Rule-governed behavior and sensitivity to changing consequences of responding. Journal of the Experimental Analysis of Behavior, 45(3), 237-256.

Hunziker, M.H.L., Caramori, F.C., Silva, A.P., \& Barba, L.S. (1998). Efeitos da historia de reforçamento sobre a variabilidade comportamental. Psicologia: Teoria e Pesquisa, 14(2), 149-159.

Kaufman, A., Baron, A., \& Kopp, R.E. (1966). Some effects of instructions on human operant behavior. Psychonomic Monograph Supplements, 1(11), 243-250.

LeFrancois, J.R., \& Metzger, B. (1993). Lowresponse-rate conditioning history and fixed-interval responding in rats. Journal of the Experimental Analysis of Behavior, 59(3), 543549.

Lowe, C.F. (1979). Determinants of human operant behaviour. In M. D. Zeiler \& P. Harzem (Eds.), Reinforcement and the organization of behavior (pp. 159-192). New York: John, Wiley \& Sons.

Lowe, C.F., Beasty, A., \& Bentall, R.P. (1983). The role of verbal behavior in human learning: Infant performance on fixed-interval schedules. Journal of the Experimental Analysis of Behavior, 39(1), 157-164.

Mace, F.C., Mauro, B., Boyajian, A.E., \& Eckert, T.L. (1997). Effects of reinforcer quality on behavioral momentum: Coordinated applied and basic research. Journal of Applied Behavior Analysis, 30(1), 1-20. 
Matos, M.A. (2001). Comportamento governado por regras. Revista Brasileira de Terapia Comportamental e Cognitiva, 3(2), 51-66.

Nevin, J.A., Mandell, C., \& Atak, J.R. (1983). The analysis of behavioral momentum. Journal of the Experimental Analysis of Behavior, 39(1), 49-59.

Okouchi, H. (2002). Individual differences in human fixed-interval performance. Psychological Record, 52(2), 173-186.

Paracampo, C.C.P., \& Albuquerque, L.C. (2004). Análise do papel das conseqüências programadas no seguimento de regras. Interação em Psicologia, 8(2), 237-245.

Porto, T.H., Ramos, M.N., \& Costa, C.E. (2011). História de aquisição do comportamento em um múltiplo FR-DRL: Diferenciação e estabilidade das taxas de respostas. Acta Comportamentalia, 19(3), 281-306.

Rosenfarb, I.S., Newland, M.C., Brannon, S.E., \& Howey, D.S. (1992). Effects of self-generated rules on the development of schedule-controlled behavior. Journal of the Experimental Analysis of Behavior, 58(1), 107-121.

Shimoff, E., Catania, A.C., \& Matthews, B.A. (1981). Uninstructed human responding: Sensitivity of low-rate performance to schedule contingencies. Journal of the Experimental Analysis of Behavior, 36(2), 207-220.

Skinner, B.F. (1969). Contingencies of reinforcement. New York, NY: Appleton-Century-Crofts.

Skinner, B.F. (1974). About behaviorism. New York, NY: Vintage Books.

Wanchisen, B.A. (1990). Forgetting the lessons of history. The Behavior Analyst, 13(1), 31-37.

Wanchisen, B.A., Sutphin, G.E., Balogh, S.A., \& Tatham, T.A. (1998). Lasting efects of a behavioral history of low-rate responding in rats. Learning and Motivation, 29, 220-235.

Weiner, H. (1964). Conditioning history and human fixed-interval performance. Journal of the Experimental Analysis of Behavior, 7(5), 383-385.

Weiner, H. (1965). Conditioning history and maladaptative human operant behavior. Psychological Reports, 17(3), 935-942.

Weiner, H. (1969). Controlling human fixed-interval performance. Journal of the Experimental Analysis of Behavior, 12(3), 349-373.
Weiner, H. (1970). Human behavioral persistence. Psychological Record, 20(4), 445-456.

Weiner, H. (1983). Some thoughts on discrepant human-animal performances under schedules of reinforcement. Psychological Record, 33(4), 521-532.

Wulfert, E., Greenway, D.E., Farkas, P., Hayes, S.C., \& Dougher, M.J. (1994). Correlation between self-reported rigidity and rule-governed insensitivity to operant contingencies. Journal of Applied Behavior Analysis, 27(4), 659-671.

\section{Informações do Artigo}

Histórico do artigo:

Submetido em: 04/03/15

Primeira decisão editorial: 17/04/15

Aceito em: 24/06/15

Editor Associado: Edson M. Huziwara 